2. That general or systemic symptoms are often obviously recognizable, and occasionally are severe.

3. That we should distinguish between erythema multiforme and other forms of exudative erythema, not every exudative erythema being an erythema multiforme.

4. The term erythema multiforme should be confined in its employment to characteristic inflammatory manifestations, especially of the superficial strata of the skin, and that where pronounced hemorrhagic lesions occur in the integument, as well as elsewhere, the disease should be viewed as purpura.

5. Erythema multiforme is probably not infre quently an infectious disease, both the general and local symptoms pointing to this conclusion.

$$
\text { DISCUSSION. }
$$

Dr. BAUM-I have seen both varieties of erythema multi forme on the same patient, $i . e .$, the erythematous variety on the upper extremities and the hemorrhagic lesions on the legs. I think the disease due to a vasomotor disturbance. I recall a case of this disease in which there were present alternating bullous and hemorrhagic lesions. A relapse had occurred after the death of a sister and it presented the large bullous variety in the hands. I think there were lesions also in the bowels, because there was marked irritability there.

Dr. BulkLeY - I consider it an infectious disease and due to an auto-intoxication. I have seen many cases of this disease in recently landed immigrants. I advise the administration of Startin's mixture and a calomel purge. The rheumatic element occurs sometimes and I regard it as another symptom.

Dr. HaRTZELL-All the cases classed under this disease do not belong there. Erythema multiforme exhibits special features of an infectious disease. There are joint affections which are rheumatoid in character, but it is not rheumatism. I do not think the course of the disease is at all influenced by treatment. A number of cases are due to various poisons, e.g., drugs, but the lesions should not be classed as erythema multiforme.

Dr. Pollitzer--The cutaneous lesions are only symptome of a general disease and the affection is due to some disturbance of the blood vessels. Pathologically there is an erythema accompanied by an exudate and the hemorrhagic form is only one of degree. I do not think it correct to classify the purpura which sometimes follows the ingestion of quinin, with the thrombosis of endocarditis.

Dr. Cook-1 think erythema multiforme a group of diseases due to neurotic influences.

Dr. GiLCHRIST-I consider the cutaneous lesions are prob ably the expression of a general affection. I have examined numbers of microscopic sections taken from four or five cases of the ery thematous variety of thie disease and also from one case of the bullous form. The results of my observations seemed to point to the fact that the cutaneous lesions were brought about by some-toxin or some micro-organisms which could not be demonstrated, forming or developing directly beneath the epidermis. The polynuclear leucocytes wandered out into this region and appeared, in the vesicular variety, to become disin tegrated or killed as soon as they had wandered out. This condition seemed to point to the fact that the poison was a powerful one. This remarkable microscopic picture could not be explained by any neurotic influences, nor by any alteration in the vessels. The bullous and hemorrhagic varieties of the disease were only one of degree.

With reference to immigrant dermatitis, a good deal of experience was had with this affection in Baltimore, since it was a considerable port of entry for immigrants. I would not class this group with erythema multiforme but would consider it as a separate affection. In the treatment of erythema multiforme I have found sodium salicylate very efficacious.

Dr. Duhring-Most of the speakers agree with me that there is more in this disease than appears on the surface. I consider Hebra's definition, which was given thirty-five years ago, just as applicable today and could not be improved upon. Hebra, however, did not touch upon the constitutional symptoms. Erythema multiforme is not hemorrhagic. Although the causes are identical, yet all the varieties are not infectious. Immigrant dermatitis I would call ery thema exudativum. The cases of erythema multiforme I have seen in Philadelphia are milder than those seen in New York, London, etc.

\section{ON VARIOUS FORMS OF CUTANEOUS TUBERCULOSIS.}

Presented to the Section on Cutaneous Medicine and Surgery, at the Forty-eighth Annual Meeting of the American Medical

Association, held at Philadelphia, Pa.. June 1-4, 1897.

BY A. RAVOGLI, M.D.

CINCINNATI, OHIO.

The affections of the skin produced by tuberculosis have shown such a variety of form that they offer nearly the same interest as those arising from syphilis. On account of the complex construction of the skin the tubercular lesions must present a different appearance according to the organs and tissues of the skin involved. When the glands of the skin are affected we see lichen scrofulosorum or folliculitis; when the lymphatics are affected, the disease manifests itself in the form of tubercular gumma, and as verrucous cutaneous tuberculosis, or lupus, when the papillary layer or the derma is invaded.

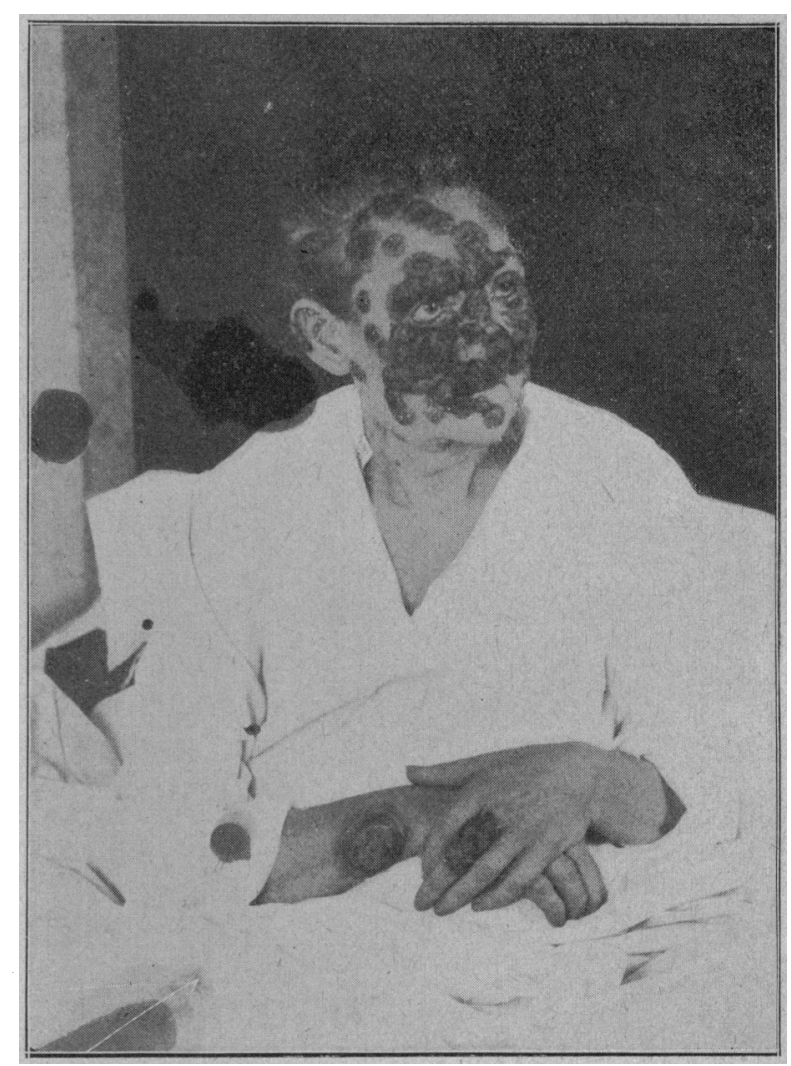

Figure 1.

The cause of these eruptions is the tubercle bacillus, which is found in the structure of the skin. We have, however, other cutaneous eruptions where no tubercle bacilli are present, and they are the result of its toxic products. The toxic power of the tox-albumins has been already demonstrated, and it gives the explanation of those forms of lupus erythematosus and of lupus pernio of Hutchinson. In these cases the tubercle bacillus may be in the internal organs far from the skin or in the mucous membranes near the affected skin, and the toxins are the cause of the eruption which is only symptomatic and persistent on account of the persistency of its cause.

The most superficial form of tubercular erythema is considered a serious symptom for the future of the patient. Erythematous eruptions in form of roseola 
remaining from twenty to thirty days have been observed by Bayet and Schlangreieff, in individuals affected with acute pulmonary tuberculosis. Last year I attended a lady with a superficial form of lupus erythematosus of the face and hands, suffering with advanced pulmonary tuberculosis. The eruption on the face appeared two months after the pulmonary symptoms became manifest remaining for over one year and when the pulmonary symptoms increased in severity, the eruption had nearly disappeared.

Those forms of cutaneous tuberculosis, in whioh the tubercle bacillus and the tubercular process is present in the skin, are the subject of this paper. In many of these cases we find the individual in good general health, in spite of the cutaneous ulcerations. Some of these forms are the result of auto-inoculation, the tubercle bacilli from the same person inoculating the skin, others are the result of outside inoculation as in verruca necrogenica.

H. Hallopeau ${ }^{1}$ calls these forms tuberculoses cutanées bacillaires in order to indicate that they are the

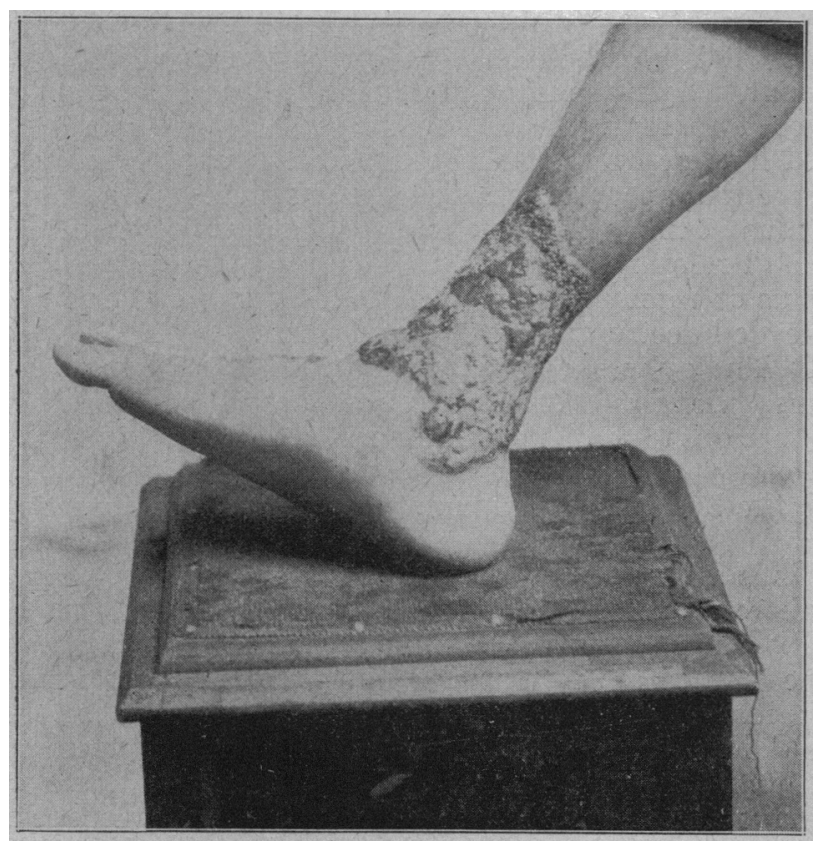

Figure $2 a$

direct result of the presence of the tubercle bacillus in the skin. In these cases we have the alterations in the papillary layer with accompanying lesions of the epidermis and often also of the derma. The lesions of the papillary layer take on a vegetating form, for which reason they were called by Riehl and Paltauf, tuberculosis verrucosa, or tuberculosis papillomateuse by Besnier, Deboue and Brissaud.

The illustrations are of three cases of cutaneous tuberculosis of this kind, where the infection came directly from the same individual, and in each the affection showed a different course and appearance.

Figure 1 shows an extensive tubercular eruption in a lady 52 years old, suffering with pulmonary tuberculosis for over three years, accompanied with profuse expectoration. The eruption has now completely disappeared and only superficial scars show the spots formerly occupied by the eruptive plaques.

1 Sur le rapports de la tuberculose avec les maladies de la peau, autres qui le Lupus Vulgaire, Third International Congress of Dermatology,
The examination of the sputa revealed a large number of tubercle bacilli. The eruption began on the extensor surface of the right arm near the wrist joint, with an intense itching and an erythematous patch which became somewhat swollen and was soon covered with small pustules, forming dirty brown crusts. While the pustules were spreading superficially in the middle, ulcerations formed which gradually gave place to vegetating granulations, showing the verrucous appearance. Almost contemporaneously patches of the same nature affected the lips, spread upon the nose, cheeks and forehead, scalp and both sides of the neck. On account of the itching sensation she often rubbed the spots and the other hand was inocculated on the dorsal surface of the third finger in the form of a large eruptive spot with the same characteristics as the spots on the face. From cut one can easily see the three zones described in the typical patches; the outer zone erythematous, slightly raised above the level of the normal skin, the middle zone, which consists of a dusky livid infiltration with scattered ulcerations, and small abscesses, somewhat

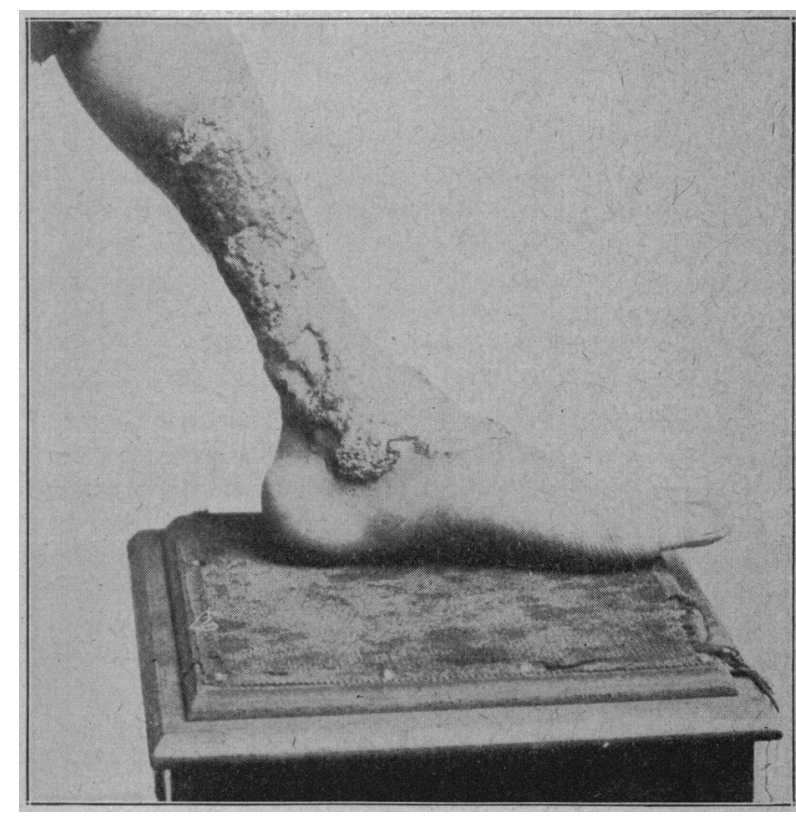

Figure $2 b$.

raised above the former; the inner zone shows fissures and sprouting up from them are small warty projections making this zone somewhat higher than the others.

I was not allowed to make microscopic examinations of the case, but the attending physician asserted that he found tubercle bacilli in the secretion of the ulceration. The most remarkable feature in this case was the relatively acute course and the recovery. The eruption lasted from three to four months, recovering gradually like any other superficial ulceration, leaving $a$ red parchment-like scar. The lady has also improved considerably in her general health.

Figure 2 is another case of tuberculosis verrucosa outis, which lasted over four years in spite of strong and constant treatment. At present the ulcerations have nearly all healed. The patient is a man of strong physique, molder by trade, has always enjoyed good health, never had syphilis. Five years ago he had an abscess on the internal region of the left leg 
with caries of the tibia. In the cut an irregular scar in this region shows it plainly, and at this point was the beginning of the cutaneous ulceration. It spread from the edges of the wound and gradually extended, covering the whole extent of the limb, to the dorsal surface of the foot. At the same time another abscess of the same nature, with caries of the bone affected the right leg. Under surgical treatment the bone healed, the surface was covered with granulations, but no healthy scar could be obtained. The ulceration spread with exuberant vegetations, encircling the whole calf of the leg, reaching the opposite side in the malleolar and tarsian region. The vegetations were in the form of a mass elevated above the level of the normal skin, easily bleeding in some places, covered with crusts, in others with thick epidermic masses. The detritus was often examined for the tubercle bacillus, but it was never found. In a few sections the epidermis was much thicker, red and of verrucous appearance, the papillæ greatly enlarged, with an enormous infiltration of small round cells, in some places arranged in foci, resembling the tissues affected with tuberculous inflammation. In some, few giant cells could be found surrounded by numerous nuclei of a tubercular character.

In this year after a tonic internal treatment the patient has gained considerably in flesh. The patches have been cauterized with Vienna paste, in other places have been curetted, and gradually with the application of a resorcin and creosote salve the ulcerated surface began to appear more healthy and had nearly all healed, leaving scars and an atrophic condition of the affected regions.

Bacillar infection may be carried from the lymphatic glands to the skin as we often see in the scrofuloderma. The same infection can at times be spread from the inguinal glands to the skin and surrounding tissues in the form of phagedenic bubo. Figure 3 shows a young colored man with an enormous ulceration of the right groin spreading down to the internal region of the thigh, on the penis and on the scrotum, and another beginning on the other groin.

He is 20 years old; his father is living and in good health; his mother died of consumption. $\mathrm{H}_{\theta}$ has been affected with scrofula, and the condition of the cervical glands, swollen and covered with scars, attests it. $\mathrm{He}$ was admitted to the hospital for inguinal bubo. He stated that nearly eight weeks before entering the institution he noticed a small ulceration at the end of prepuce. A few days after there appeared a swelling of the left groin. The skin soon became tense and painful, broke down and discharged a foul sanguinolent and purulent fluid. $\mathrm{He}$ stated that the sore on the prepuce appeared two months after the last intercourse. When he was admitted Nov. 7, 1896, he appeared to be well developed and nourished, only his neck showing many scars of old tubercular glands. Both lungs gave mucous râles at the apex in prolonged expiration, no dulness. The body was free from eruption, no sore throat, no alopecia. The right inguinal region showed a swelling of about the size of the fist, two small openings gave issue to some blood and pus. The base of the swelling was hard for some distance. Glands of left groin were also enlarged. There was a small, hard, superficial ulceration on the free end of the prepuce. Under chloroform the right groin was opened, thoroughly curetted and packed with iodoform gauze. Instead of obtaining cicatrization, the whole surface began to show a large ulcerated surface, with fungous growths affecting the inguinal region to the internal region of the thigh, the scrotum riddled with sinuses, the skin of the penis showed hard lymphatic vessels like small gummæ, which gradually formed abscesses and ulcerations with the same fungous appearance. The left inguinal glands were hardened and formed a large mass with points of suppuration beneath.

February 1 the patient was again placed under the anesthetic; the sinuses were opened, the glands removed, the granulations thoroughly curetted and the whole surface was cauterized with the thermo-cautery. Succeeding dressings were made with acetanilid. Although no symptoms of constitutional syphilis had so far appeared, yet liquor Van Swieten had been administered, together with tonics and reconstituents. Mercurial plaster had been used on the ulcerations without result. In order to observe the reaction to tuberculin, injections were given of from 1 to $5 \mathrm{mgr}$., and at every injection he had fever ranging from 101 to 103 degrees $F$. When I went out of service the ulceration was somewhat healed, the granulations appeared healthier and the patient was able to go about. Symptoms of pulmonary tuberculosis were more apparent.

I have referred to this case as an example of mixed infection, and from many cases of phagedenic ulcers I must come to the conclusion that tuberculosis of the glands and lymphatics is often mixed with the virus of chancroid or syphilitic lesions, producing the dreaded destructions of phagedenism.

This boy was affected with tubercular glands, and when the glands broke down on account of the other infection, tubercle bacilli came in contact with the ulcerated surface, causing a tubercular ulcer in place of the bubo. The gummous tuberculosis is in many instances the cause of the cutaneous tubercular ulcers; this is a kind of intermediary between the deep-seated tuberculosis and the superficial of the skin. In all tubercular forms we must see a mixed infection, as the cocci of the pus appears in every tubercular process as secondary elements, so that we find in the middle a cheesy, degenerated point with a purulent softening, as referred to by Leistikow. ${ }^{2}$ In cases of phagedena we may have not only the staphylococcus pyogenes and the tubercle bacillus, but also the syph. ilitic virus or the micro-organism proper to chancroid, which are the commencing agents of the ulceration.

In our case it is rather difficult to establish with certainty whether the ulcer which started from the prepuce was a chancroidal or a syphilitic one. The patient after six months has not yet shown any syphilitic symptoms, with the exception of the phagedenic bubo. It is also hard to believe it a chancroid, when the patient asserts that the ulcer appeared two months after the last intercourse. It seems to me that an accidental sore of some nature, has started the inflammatory process in the lymphatic vessels of the penis and inguinal glands which, affected already with tubercular process, broke down, causing extensive abscesses, and the tubercle bacilli from the tubercular glands have infected the surrounding tissues, causing a cutaneous and subcutaneous tubercular gum. mous ulceration. The ulcers of the dorsum penis and scrotum are due to tubercular lymphangitis, as we found the affection to start directly from the lymphatic vascular trunks. They appeared at first hard 
like stems under the pressure of the finger, then they became larger, of dusky color, breaking down into a quantity of purulent and sanguinolent fluid and a great discharge of lymph. In this case two conditions could have been the cause of the process. Either the ulcer itself was of tubercular origin and infected the lymphatics, or the lymphatics were already infected with tubercle bacilli, as were the inguinal glands, and therefore the staphylococci started the inflammatory process around the already infiltrated tubercular tissues producing tubercular ulcerations with papillary growths.

I referred to these three cases of tuberculosis verrucosa cutis on account of their peculiar origin. The first was the result of the inoculation of the tubercle bacilli from the sputa; the second was produced by the tubercle bacillus from the diseased bone; the

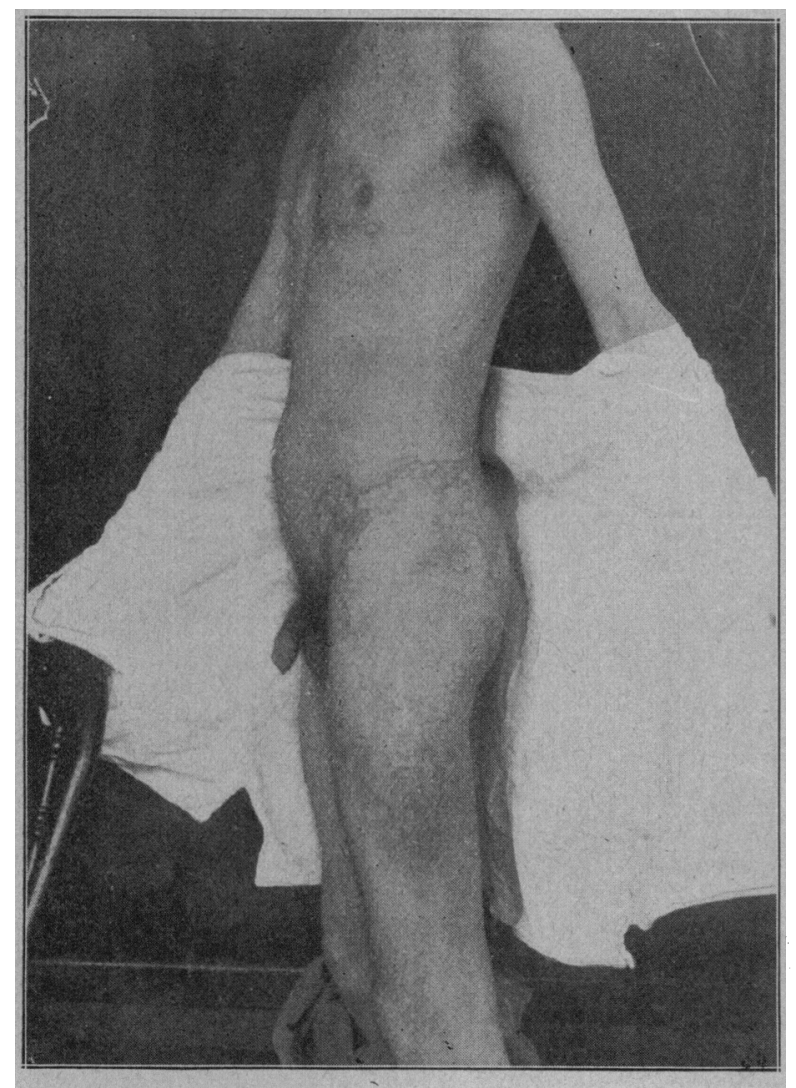

Figure 3.

third from the bacilli contained in the tuberoular glands.

Each one has some important features. The first in which the papillary layer and the epidermis only was superficially affected, healed in about four or five months, leaving superficial scars. The second which affected the derma deeply formed thick vegetating papillæ which lasted nearly four years, stubborn and persistent in spite of the surgical means employed. The third, a type of mixed infection affecting the skin in its deepest layers and the fascia, with vegetating and papillomatous appearance, equally resisted the cautery and the curetting. There is no doubt that the layers of the skin affected have much to do with the more or less persistent character of these affections.

I now call your attention to two cases, which I classify among the the scrofulo-tuberculosis, accord- ing to the distinction proposed by Maurice Vallas. ${ }^{3}$ In these cases we do not find any outside inoculation; they are bacillary in their nature like lupus, and are primary, showing no trace of their origin. They could be ranged with Leloir ${ }^{4}$ among the atypic varieties of lupus.

Figure 4 is a good specimen of this variety of cutaneous tuberculosis. It is of a man 30 years old, of good physique, blond, lumber-yard hand by occupation. No history of tuberculosis nor cancer exists in his family. He never had syphilis, has always enjoyed good health. Dec. 29, 1896, he came to the hospital for ulcerations of the skin. The eruptions had begun fifteen months previously, at first in the form of a little indurated red patch over the crest of the left ilium extending internally. It gradually spread and began to break down in the center, form-

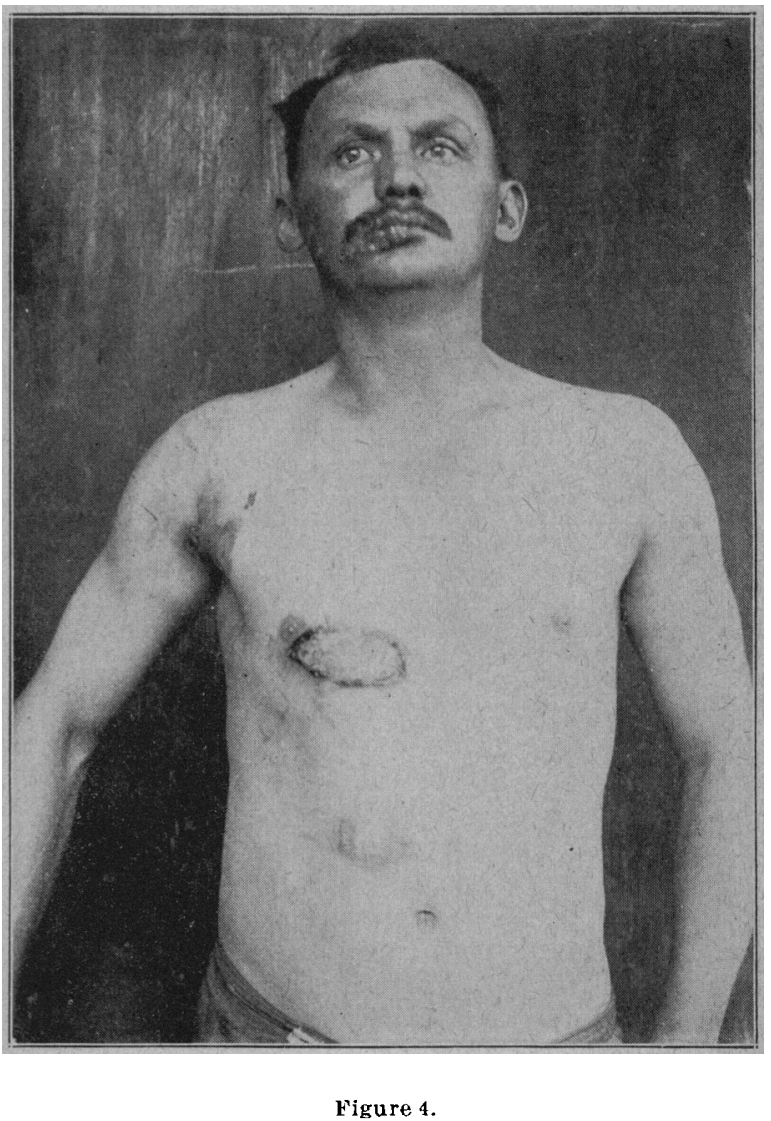

ing an irregular ulcer, tender and slightly painful. When the ulcerated patch had attained the size of a silver dollar, healing began at the center, leaving an irregular thick and superficial scar. The peripheral ulcerations continued spreading and after assuming the size of the palm of the hand, stopped and after some months healed. Other patches of the same character after an interval of two months appeared, one on the right leg near the knee, one on the abdominal wall to the right of the umbilicus, one in the right axilla and shoulder, and one on the right side of the mouth, involving both lips. The eruption consisted of nodules deeply seated in the derma and coalescent at the periphery of a thick cicatrix, resembling the lupus scars. Some of these nodules were

3 Les ulcères tuberculeuses de la peau, Thèse de Lyon, 1887. 4 Ann. de Dermat., 1888. 
aiready ulcerated and covered with thick brown crusts, and some with thick epidermic scales.

Under the microscope the corium appears affected with round foci infiltrated with small cells grouped together. The same cells are infiltrating the connective tissue, and the fascicles appear in a reticular manner separated by the cellular infiltration. Small blood vessels appear enlarged and some are plugged with infiltration, showing a kind of endo-arteritis. In some places the papillæ have been destroyed by the infiltrating elements, and where they remain appear enlarged and hypertrophic.

Tubercle bacilli were not found. No doubt this was a tubercular form of cutaneous disease, which had a great resemblance to lupus, and which we can refer to as an atypical variety of lupus.

The treatment consisted in tuberculin injections of from 1 to $2 \mathrm{mgr}$. every other day. The first injections were followed by fever and local reaction. The temperature rose three hours after the injection to $102 \mathrm{~F}$., with symptoms of general malaise, pain in the spinal region, and sometimes vomiting. On the local spots, the reaction was exceedingly marked, an intense vivid purplish color showed all over the patches and nodules, and the ulcerated spots appeared of dark brown color, as after cauterization. After the third injection the temperature rose only to 99 with very little general disturbance, but the local reaction was always marked. Locally a dressing of sterilized vaselin was used. The crusts were soon removed; the ulcers began to be covered with good granulations, and on January 9, the ulcerations having completely healed, the patient was discharged.

Figure 5 shows another superficial tubercular skin eruption of ulcerative nature, which can be referred to the same class of tuberculosis lupoides. The patient was admitted to the hospital Jan. 11, 1897, for a diffuse ulceration on the left thigh, coursing nearly the entire gluteal region and abdominal wall. Another patch of the same nature is in the left mammary region. $\mathrm{He}_{\theta}$ was 44 years old, of poor physique, had suffered a long time with pleuro-pneumonia, no history of syphilis can be found. The present eruption began three years ago as a small hard patch, somewhat elevated above the level of the normal skin which soon broke down, causing an ulceration. This enlarged in a serpiginous way, healing slowly in the center and ulcerating at the edges, until in nearly three years it had taken on the proportions referred to. The ulcerations after healing left very superficial scars, depressed in the center and atrophic, whioh gradually became very white.

The examination of the chest did not reveal marked dulness nor râles.

The affection had been treated previously as a syphilitic one with iodid of potassium and local application of mercurial plaster, with little results.

As soon as he entered the hospital he had tuberculin injected from 1 to 3 milligram. The fever was not marked, but the local reaction was very striking. The whole affected surface became a purplish red, and the ulcerated places appeared as though they had been cauterized.

In this case also, the whole treatment consisted in tuberculin injections alternate days. Elixir of iron, quinin and strychnia internally, and locally a simple dressing with sterilized vaselin.

Under the tuberculin the progress was so rapid that March 18 he left the hospital entirely recovered.
This case was somewhat different from the preceding. The infiltration was much more diffused and superficial, resembling more lupus erythematosus than lupus vulgaris, while the other was more like a case of lupus vulgaris.

The marked reaction to the tuberculin injections is an important point for the diagnosis of the tuberculosis of the skin, and the result obtained under its use is a guarantee that the affection could not be from any other cause.

Prof. V. Bergmann, at the meeting of Freien Vereinigung der Chirurgen in Berlin, November, 1890, referred to a case of tubercular ulceration of the lower jaw and palate, which healed entirely after a few injections of tuberculin 0.1,0.2. He noticed slight general but very marked local reaction on the first day.

After the tuberculin injection the tubercular nodules appeared much smaller and the ulcerations as if they had been cauterized. Dr. C. Schimmelbusch ${ }^{5}$ stated that he could not find true necrosis in the tubercles of the skin after tuberculin injections, but the tissues showed only an inflammation and an exudation process, causing a sloughing of the ulceration.

The important feature in these last cases is the curative effect of the tuberculin; only simple vaselin dressings were applied to the local ulcerations.

I have referred to these cases, which lately came under my treatment, on account of the interest which they offer to the dermatologist. The first three cases, tuberculosis verrucosa cutis, are of scientific importance on account of the source of infection, and the course and extension of the lesions. Suppuration is the principal cause of spreading of this tuberculosis and miliary foci are formed, so that the pyogenic cocci cause the extending of these eruptions. In these forms tubercle bacilli are difficult to find and Riehl and Paltauf believed the cause of this affection to be due mostly to a mixture of the infectious germs. Nearly all cutaneous tuberculoses end with ulceration; in some, however, the ulceration is a most remarkable phenomenon, showing a tendency to proliferation.

The two last cases have more resemblance to lupus, and especially the last case can be compared with lupus tuberculosus superficialis, as described by W. Dubruille at the Society of Medicine and Surgery of Bordeaux. In these cases we find small superficial tubercular gummata, which after ulcerating have a tendency to recovery, leaving a superficial scar.

In the tuberculoses of the skin, with the exception of the tubercular ulcers, which are developed near the natural orifices in advanced consumptive patients, and which have a destructive nature, the other mentioned forms usually recover. It seems that the tubercle bacillus in the skin is somewhat modified, has not much tendency to multiply, and is not easily inoculable. The derma does not offer to this bacillus a fertile ground and its infectious properties remain limited to the skin, without showing general infectious phenomena. At times the infectious power of this germ, when encapsuled in the tissues, may remain latent for years, ready to break out at any time in the form of lupus from old scars.

In all our cases we have seen recovery of the cutaneous tubercular form. In the forms of tuberculosis verrucosa, by means of curetting, the cautery and various dressings we have obtained recovery. In the

Mikroscopische Befunde der Tuberculose der Haut, und der Sichtbaren Schleimhaute nach Anwendung der Kochschen Mittels. Deutsch. Med. Woch. 1896. 
two other cases, the tuberculin injections produced beneficial influence on the tubercular cutaneous forms No other dressing was used than vaselin, and yet after several injections the ulcerations healed promptly.

I will say in conclusion, that cutaneous tuberculosis occurs not very rarely; that it is not always accompanied with general tubercular infection; that it has a tendency to recovery; that it may yield to local as well as to general treatment; that tuberculin injections for some ulcerative forms have a beneficial influence.

\section{THREE CASES OF TUBERCULOSIS CUTIS VERRUCOSA.}

Presented in the Section on Cutaneous Medicine and Surgery, at the Forty-eighth Annual Meeting of the American Medical Asso. ciation, held at Philadelphia, Pa., June 1-4, 1897.

BY M. B. HARTZELL, M.D.

INSTRUCTOR IN DERMATOLOGY, UNIYERSTY OF PENNSYLVANIA. PHILADELPHIA, PA.

Although that form of tuberculosis of the skin first described by Riehl and Paltauf, under the name of tuberculosis cutis verrucosa can no longer be regarded as a rare affection, and usually presents such well. marked and special features that its diagnosis is ordinarily made without any difficulty, I have thought that it might be of interest to report the three cases the subject of this paper, since each of them presents features, clinical and histologic, which are uncommon. The first case reported, presented in its early stages a form of cutaneous tuberculosis to which attention has not yet, so far as I am aware, been called.

Case 1.-R. L., about 40 years of age, was referred to me for advice concerning an affection of the thumb which had existed for three years, in spite of much and varied treatment. The dis ease consisted of an irregularly outlined, noticeably elevated, bluish-red patch, whose surface was covered with numerous small papillæ and scanty adherent grayish and brownish scales, occupying the greater part of the extensor surface of the ter minal phalanx of the right thumb. This wart-like patch was surrounded by a smooth violaceous border about an eighth of an inch wide, less elevated than the patch itself. The thumbnail was also considerably deformed, the imperfect growth of the nail being due to the manner in which the disease began, to which further reference will be made. On close examination a number of small openings could be seen between the papillæ which studded the surface, from which pressure caused a few drops of thick pus to escape; pus could also be pressed out from beneath the nail-fold at the root of the nail. The history of the malady, obtained from the patient, was as follows :

About three years previously the root of the nail became red, swollen and slightly painful, and remained so without material change for some months. Gradually the skin of the thumb became thickened, violaceous in color, and finally cov. ered with small papillæ from between which, in places, cheesy pus could be expressed. Pain was never at any time a prominent symptom, and the patient was annoyed quite as much by the unsightly appearance of the member as by any disagreeble sensations in it. The patient's general health was far from good ; he had a chronic cough, somewhat paroxysmal in character and accompanied by fres expectoration; he was hoarse, and had been losing flesh for some months past. The treatment consisted in the destruction of the entire patch by means of a plaster containing 40 per cent. of pyrogallol, which was followed by an ointment of resorcin-1 to 16 -until cicatrization occurred. An apparent cure was thus obtained within three or four weeks ; but two months later there was a slight relapse, two split pea-sized, brownish-red translucent nodules appearing in the cicatrix. It is of interest to note that these nodules presented the typical "apple jelly" appearance of lupus vulgaris, indicating, if this were any longer a matter of doubt, the etiologic indentity of these two clinically quite distinct forms of cutaneous tuberculosis. As to the mode of inoculation in this particular case, it was noticed that the patient was in the habit of frequently biting his thumb, unconsciously, and this habit taken in connection with the chronic cough and progressive loss of weight suggests a pos sible way in which the skin may have been inoculated. The chief point of interest, however, is the form which the malady assumed in its early stages. It began as a paronychia, and remained such for some months without showing any of the features characteristic of cutaneous tuberculosis. Some six months after coming under my care the nail-fold about the root of the left index finger became red, swollen and moderately painful; after a time, minute openings made their appearance in the swollen tissues, and a small quanity of thick pus escaped. This affection of the index finger did not differ in appearance from ordinary paronychia, but was absolutely uninfluenced by the treatment advised-application of mercu rial ointments chiefly. The patient, who was a more than ordinarily intelligent person, declared positively, that the disease of the thumb began in a precisely similar manner. I think it is evident from this case that we must include among the manifestations of cutaneous tuberculosis a form of chronic parony chia, which in its early stages at least resembles, clinically, paronychia due to other causes.

Case 2.-T. B., 44 years of age, employed by a railway company to clean freight cars, in which live stock had been trans ported, came under observation in November, 1896, having upon the back of the right hand, near the metacarpo-phalangeal joint of the index finger, a circular elevated patch in which were a number of pin-head-sized openings, some of which contained small horny plugs which could be picked out with the forceps; others gave exit to a drop of pus when pressure was made upon the lesion. This patch had existed for some months, was only slightly painful, and was slowly grow ing larger. It had followed a slight injury to the hand received by knocking it against the end of a board while cleaning a cat tle car. This trifling wound had at first promptly healed, bu later became slightly painful, swollen and gradually assumed its present aspect. The patient was in excellent general health, weighing about 175 pounds, and so far as could be learned had never been seriously ill. After his first visit the patien was not seen again for a considerable time; and upon a second later examination the patch was seen to have undergone a nota ble alteration in its appearance. Beside having increased in size, its surface had become verrucose, and the horny plugs spoken of had disappeared. The diagnosis of tuberculosis cutis having been made, the lesion was destroyed by the appli. cation of a strong pyrogallol plaster for ten days, a 20 per cent. resorcin plaster being afterward employed. Within one month a complete and permanent cure was obtained. The patient was seen one year later and the hand was perfectly sound; but his general health had changed very greatly for the worse. He was emaciated; had a persistent and frequen cough, accompanied by abundant expectoration; his midday temperature at the time of this examination was $101.5 \mathrm{~F}$. Examination of his chest revealed decided dulness at the left apex with numerous râles.

In their paper published in 1886 , Riehl and Paltauf called attention to the resemblance, in some cases, between their tuberculosis cutis verrucosa and the "perifolliculite suppurée et conglomerée en placard" described by Leloir; and in the case just reported the resemblance to this form of follicultis was so marked that the diagnosis was for a time in doubt. In the more recent descriptions of this form of tuberculosis by other authors, little or no mention is made of the possibility of confounding these two affections; but the resemblance may, at times, be so close as to make the possibility of error consid erable. A microscopic examination of an excised portion of the patch in this case showed that the follicles were markedly involved in the morbid process, there being numerous miliary abscesses in the perifollicular parts which communicated in some cases with the follicle. The macroscopic and microscopic appearances justify the assumption that the malady began as a perifolliculitis, and later invaded the interfollicular tissues.

Case 3.-The last case I report-in some respects the most interesting of the three-is unfortunately very defective in its clinical details, since I have been able to procure but very few facts as to its history, which is as follows:

Mrs. F., aged 67, had a circular, considerably elevated, wartlike lesion, the size of a quarter, which had existed for about one year upon the flexor surface of the fore-arm, about two inches above the wrist. This, according to her statement, had begun as a small " pimple," which steadily enlarged and became covered with numerous enlarged papillæ. The entire lesion, together with a portion of the surrounding tissues, was excised and submitted to me for microscopic examination. The excised lesion presented the macroscopic appearances of a verrucose tuberculosis, but having been in alcohol for some days before coming into my hands, a positive diagnosis from the naked eye appearances was somewhat difficult. Examination 